\title{
Factores que influyen en el uso de teléfonos móviles en el contexto de aprendizaje por parte del profesorado de educación superior en la provincia de Santa Catarina, Brasil
}

\author{
MARIA JOSÉ CARVALHO DE SOUZA DOMINGUES* \\ PÉRICLES EWALDO JADER PEREIRA** \\ JÉFERSON DELEON FÁVERO***
}

Artículo de investigación sobre la influencia del uso de teléfonos móviles en el contexto de aprendizaje.

Recibido: 1 de mayo de 2020 - Evaluado: 3 de agosto de 2020 - Aceptado: 16 de septiembre de 2020

Citar como: Carvalho de Souza, M. J., Jader, P. y Fávero, J. (2021). Factores que influyen en el uso de teléfonos móviles en el contexto de aprendizaje por parte del profesorado de educación superior en la provincia de Santa Catarina, Brasil. Hallazgos, 18(35), 181-206. https://doi. org/10.15332/2422409X.5773

\footnotetext{
* Doctora en Ingeniería. Profesora titular del Programa de Administración y Contabilidad de la Universidad Regional de Blumenau, Santa Catarina, Brasil.

Correo electrónico: mariadomingues@furb.br ORCID: https://orcid.org/0000-0001-7771-144X

** Magíster en Administración y profesor de la Uniasselvi, Brasil. Correo electrónico: peckjader@gmail.com ORCID: https://orcid.org/0000-0002-4407-7398

*** Magíster en Administración y profesor de la Uniasselvi, Brasil. Correo electrónico: jeferson_deleon@hotmail.com ORCID: https://orcid.org/0000-0002-6816-201X
} 


\section{Resumen}

El objetivo de este artículo es evaluar los factores que influyen en el uso de teléfonos móviles en el contexto de aprendizaje por parte del profesorado de educación superior. Se realizó una investigación descriptiva con la ayuda de una encuesta cuantitativa. Los datos se obtuvieron por medio de un cuestionario basado en el modelo UTAU T 2, aplicado a docentes de educación superior en la provincia de Santa Catarina, sur de Brasil. El modelo uTAUT 2 está formado por siete variables independientes: condiciones facilitadoras, expectativas de rendimiento, expectativas de esfuerzo, influencia social, motivación hedonista, precio/valor y hábito, además de la dimensión intención de uso, caracterizada como variable dependiente. Para el análisis de los datos se empleó la regresión lineal múltiple. Los resultados muestran la motivación hedonista, la expectativa de rendimiento, la condición facilitadora, el precio/valor y el hábito como factores que influencian la intención de uso de los dispositivos móviles en el público investigado. El precio/valor se reveló como la dimensión que ejerce mayor influencia en la intención de uso de los dispositivos móviles por parte del docente en clase.

Palabras clave: Aprendizaje; Dispositivos móviles; Educación superior; Modelo UTAUT 2; Tecnologías de la información y la comunicación. 


\title{
Factors influencing the use of cell phones in the context of learning by higher education professors in the province of Santa Catarina (Brazil)
}

\begin{abstract}
The purpose of this article is to evaluate the factors that influence the use of cell phones in the context of learning by higher education professors. A descriptive research was carried out with the help of a quantitative survey. The data was obtained through a questionnaire based on the UTAUT 2 model, applied to higher education professors in the province of Santa Catarina, southern Brazil. The UTAUT 2 model is made up of seven independent variables: facilitating conditions, performance expectancy, effort expectancy, social influence, hedonic motivation, price value and habit, in addition to the intention to use dimension, characterized as a dependent variable. Multiple linear regression was used for data analysis. The results show hedonic motivation, performance expectancy, facilitating condition, price value, and habit as factors influencing the intention to use of mobile devices in the researched audience. Price value was revealed as the dimension that most influences the intention to use of mobile devices by professors in class.
\end{abstract}

Keywords: Learning; Mobile devices; Higher education; u TAU T 2 model; Information and communication technologies.

\section{Fatores que influenciam o uso do celular no contexto de aprendizagem por parte dos docentes de ensino superior em Santa Catarina, Brasil}

\section{Resumo}

O objetivo deste artigo é avaliar os fatores que influenciam o uso do celular no contexto de aprendizagem por parte dos docentes de ensino superior. Foi realizada uma pesquisa descritiva, apoiada em uma pesquisa quantitativa. Os dados foram obtidos por meio de um questionário baseado no modelo UTAU T 2, aplicado a docentes de ensino superior no estado de Santa Catarina, Sul do Brasil. O modelo UTAUT 2 está formado por sete variáveis independentes: condições facilitadoras, expectativas de desempenho, expectativas de esforço, influência social, motivação hedonista, preço/valor e hábito, além da dimensão intenção de uso, caracterizada como variável dependente. Para analisar os dados, foi utilizada a regressão linear múltipla. Os resultados mostram a motivação hedonista, a expectativa de desempenho, a condição facilitadora, o preço/valor e o hábito como fatores que influenciam a intenção de uso dos dispositivos móveis no público pesquisado. O preço/valor se demonstrou como a dimensão que exerce mais influência na intenção de uso do celular por parte dos docentes durante a aula.

Palavras-chave: Aprendizagem; Dispositivos móveis; Ensino superior; Modelo UTAUT 2; Tecnologias da informação e da comunicação. 


\section{Introducción}

Desde la década de los noventa, diversas investigaciones y modelos de aceptación y uso de la tecnología predominan en varias áreas de la sociedad, incluso en la educación. Estar “conectado” forma parte de la rutina de las personas, ya sea por la necesidad de mantenerse actualizado, por demandas profesionales, educacionales o personales (Mariano et ál., 2016). Actualmente, en el ambiente educacional, profesores y alumnos se conectan por medio de móviles, lo que facilita sus actividades e impone un perfil diferente de socialización del individuo, posterior a la ascensión tecnológica (Alves, 2018; Humanante-Ramos, García-Peñalvo y Conde-González, 2017; Torrezan y Behar, 2009).

Venkatesh et ál. (2003) sobresalen por perfeccionar un conjunto de modelos sobre la incorporación y uso de tecnologías. En 2003 surgió el modelo UTAUT, y en 2012, el utATut 2, constituido como base teórica de esta pesquisa para la construcción del objetivo de estudio: evaluar los factores que influyen en el uso de teléfonos móviles en el contexto de aprendizaje por parte del profesorado de educación superior.

El estudio se justifica por aspectos teóricos y empíricos. Teóricos, por tratarse de un universo actual para investigar, dado que los móviles son poco difundidos en clase por parte de los docentes, lo cual debe ser visto como un paradigma que debe superarse (Martins et ál., 2018), y que es necesario que la academia lo interprete como un área en la que se necesita avanzar en investigación. Empíricamente los resultados posibilitarán la creación de herramientas procesuales para los docentes y las instituciones que deseen perfeccionar estrategias y prácticas pedagógicas inherentes al proceso de enseñanza-aprendizaje.

La investigación consta de cinco secciones: la primera corresponde a la introducción. En la segunda se presentan los conceptos de aceptación de las tecnologías de la información y la comunicación (TIC) y los relacionados con los móviles en la educación y el modelo utilizado. En la tercera se demuestra el delineamiento metodológico. En la cuarta se presentan los análisis de la pesquisa. Por último, en la quinta sección, se exponen las consideraciones finales y las limitaciones del estudio. 


\section{Fundamentación teórica}

\section{Apertura tecnológica en la educación}

La convivencia de la sociedad con las tecnologías ha sido cada vez más notable, ya sea en el ambiente profesional, personal o educacional (Angeluci, Soares y de Azevedo, 2018; Martins et ál., 2018; Barreto, 2018). El contexto educacional de otro tiempo, entendido exclusivamente por el uso de la tiza y la pizarra, se presenta hoy con nuevas herramientas capaces de viabilizar el proceso de enseñanza y aprendizaje a través de pizarras interactivas, computadores portátiles, celulares o tabletas. Un nuevo escenario que ofrece a los involucrados posibilidades de reinventar, remodelar o inclusive innovar métodos y técnicas que dinamizan la escolarización (Coll y Monereo, 2010; Gomes, 2008).

Según Fernandes et ál. (2012), el advenimiento de las TIC trajo nuevas perspectivas en relación con la forma y el alcance de la educación a distancia (DE). En Brasil, el e-learning parece ser un camino sin retorno. Una de las formas recientes de potenciarlo es mediante el uso de dispositivos móviles, modalidad conocida como aprendizaje móvil o, en inglés, mobile learning (m-learning).

Para Angeluci, Soares y Azevedo (2018), el creciente uso de TIC, especialmente con la utilización de dispositivos móviles, ha modificado el comportamiento de las personas (Medeiros y Ventura, 2007; Martins et ál., 2018) y de grupos de estas en diversos aspectos, como en la forma de interactuar y de comunicarse. Es decir, se trata de usuarios que tienen en la palma de su mano dispositivos que posibilitan operaciones básicas y que manejan en tareas o actividades específicas (Barreto, 2018).

Independientemente del universo de posibilidades que las TIC proporcionan a las personas en el ambiente escolar, su impacto se percibe de manera modesta (Unesco, 2013). Aunque la Unesco (2013) definió, por medio de directrices y acciones, la introducción en las clases del aprendizaje móvil, se aprecia un escenario real distorsionado. Las instituciones de enseñanza, en su mayoría, aún están culturalmente atadas a las tecnologías y las metodologías tradicionales, y por ende rechazan las posibilidades de inmersión que los móviles facilitan al universo educativo (Unesco, 2013; Palfrey y Gasser, 2011; Torrezan y Behar, 2009).

Por su parte, Bautista, Borges y Flores (2010) indican que gracias a las TIC los métodos de enseñanza, la estructura escolar, las matrices curriculares, las atribuciones y el papel del alumno y del profesor han venido mutando constantemente, y la necesidad de adaptación a este nuevo momento es desafiante y necesaria (Arruda y Puentes, 2011; Palfrey y Gasser, 2011). 


\section{Uso de dispositivos móviles en el ambiente de enseñanza (m-learning)}

El surgimiento tecnológico da cuenta de varias alteraciones en la conducta de alumnos y docentes dentro del ambiente escolar en el transcurso de los últimos años. El m-learning puede describirse como una modalidad de enseñanza que, mediante redes inalámbricas, utiliza dispositivos móviles para facilitar: 1) la transmisión de información; 2) el acceso al contenido; 3) la interacción entre estudiantes y profesores, y 4) la consulta y el intercambio de materiales diversos en cualquier lugar y momento (Pina et ál., 2016).

El $m$-learning se define por la experiencia de aprendizaje personalizada que se brinda a cada estudiante (Traxler, 2011). El aprendizaje puede ser personalizado, ya que el m-learning permite a los estudiantes considerar la diversidad, la individualidad y los diferentes estilos de aprendizaje de los estudiantes, respetando el tiempo y el lugar de estudio (Pina et ál., 2016).

Básicamente, el m-learning emplea la movilidad tecnológica que brindan los dispositivos móviles inalámbricos portátiles, como teléfonos inteligentes, tabletas, asistentes digitales personales (PDA, por las siglas en inglés de personal digital assistants) y computadoras de mano para realizar actividades en el modo a distancia. Entre los dispositivos móviles, el teléfono inteligente es considerado el más popular, especialmente entre jóvenes y adultos, como parte de la nueva sociabilidad humana (Rocha, Joye y Moreira, 2020).

En lo que se refiere al empleo de dispositivos móviles en clase (m-learning), no hay diferencia (Alves, 2018; Torrezan y Behar, 2009). La modalidad de enseñanza tradicional ha perdido fuerza ante la convivencia de los estudiantes con los móviles, lo que ha traído consigo una nueva perspectiva de la vida dentro y fuera de la escuela (Martins et ál., 2018).

De acuerdo con Martins et ál. (2018) y Marçal, Andrade y Rios (2005), los individuos de la escuela de hoy son totalmente diferentes. El estudiante actual posee un perfil inquieto, interrogador e informado, y no mantiene la atención en el profesor y la pizarra por cuatro o cinco horas. Esta circunstancia les plantea a los docentes una ruptura de paradigma, la necesidad de implementar cambios de comportamiento y la explotación de diferentes prácticas pedagógicas, con el objetivo de adecuarse a la condición real del ambiente de enseñanza y al actual universo educativo (Palfrey y Gasser, 2011; Barcelos, Tarouco y Bercht, 2009; Kynäslahti y Seppälä, 2003). 
Para Rocha, Joye y Moreira (2020) la tecnología móvil e inalámbrica apoya el intercambio de información, el intercambio de ideas y experiencias, la resolución de dudas y el acceso a una amplia gama de recursos y materiales didácticos, que incluyen texto, imágenes, audio, video, libros electrónicos, artículos, noticias on line, contenidos de blogs, microblogs, juegos y ambientes virtuales de aprendizaje, con el fin de interactuar con compañeros y profesores en diferentes contextos. Por otro lado, se plantean numerosos desafíos, posibilidades y problemas relacionados con su implementación.

La necesidad de diferentes prácticas pedagógicas constituye un desafío para la enseñanza y principalmente para el docente (Martins et ál., 2018; Palfrey y Gasser, 2011). El espectro de cambios va desde el reto que supone la estructura escolar hasta la formación y recualificación para el uso de tecnologías. Esto se debe al perfil de la nueva generación de estudiantes (Medeiros y Ventura, 2007; Bautista, Borges y Flores, 2010) que está cada vez más conectada e informada, y que desea incluir metodologías más activas como instrumentos de aprendizaje. El alumno no desea apenas aprender los temas; su objetivo, además de internalizar el conocimiento de forma colaborativa, es ser guiado por medio de la orientación del profesor (Martins et ál., 2018; Palfrey y Gasser, 2011; Kynäslahti y Seppälä, 2003).

Por lo que atañe al proceso colaborativo y de intercambio en el modelo de enseñanza-aprendizaje, Kynäslahti y Seppälä (2003) consideran que este método favorece el procesamiento del conocimiento por el alumno, pues comunicarse, compartir y trabajar en forma colaborativa son acciones que pueden concretarse en cualquier lugar o momento. De esta forma, los estudiantes analizan problemas, exploran ideas, desarrollan conceptos, evalúan soluciones de aprendizaje, etc.

Matoski et ál. (2020) realizaron un estudio cuyo objetivo fue caracterizar el uso de dispositivos móviles como herramientas de aprendizaje. El análisis se desarrolló con profesionales y estudiantes de pregrado y posgrado. Como resultado, pudo observarse que entre las diversas herramientas utilizadas en los dispositivos móviles, las que se caracterizan por la rapidez y facilidad de acceso son las más relevantes. Sin embargo, dificultades como el miedo al robo y la actualización del dispositivo inhiben parcialmente este tipo de acceso.

Ante la importancia del uso de dispositivos móviles en la enseñanza por parte de los profesores, la Unesco creó en 2013 una guía con 13 temas que presentan las ventajas y beneficios de la utilización de dispositivos móviles en clase: 
- Expandir el alcance y la equidad de la educación.

- Facilitar el aprendizaje individualizado.

- Proporcionar retorno y evaluación inmediatos.

- Permitir el aprendizaje en cualquier momento y lugar.

- Garantizar el uso productivo del tiempo en clase.

- Crear nuevas comunidades de estudiantes.

- Apoyar el aprendizaje fuera de la clase.

- Potencializar el aprendizaje sin solución de continuidad.

- Crear un puente entre el aprendizaje formal y el no formal.

- Minimizar la interrupción educacional en áreas de conflicto y desastre.

- Auxiliar estudiantes con deficiencias.

- Mejorar la comunicación y la administración.

- Mejorar la relación coste-eficiencia.

Martins et ál. (2018) corroboran las directrices de la Unesco (2013), y expresan que el dispositivo móvil es una valiosa herramienta de apoyo para los profesores, un excelente instrumento para entender el nuevo tipo de alumno. No obstante, subrayan que este universo impone desafíos a los docentes.

Para Barreto (2018) y Lévy (2011), los desafíos del docente en el manejo de dispositivos móviles y de las tecnologías en general en el ambiente escolar están pautados por el tradicionalismo. Normalmente, el profesor no recurre a este método de enseñanza, ya que no es la forma en que él fue escolarizado y formado; es decir: él se ubica en la calidad de detentor del conocimiento y los alumnos en la de oyentes (Martins et ál., 2018; Barreto, 2018; Imbernón, 2011).

El docente, al articular la enseñanza con móviles, permite la mejoría del aprendizaje del alumno. Sin embargo, Martins et ál. (2018) y Barreto (2018) insisten en que el obstáculo en boga apunta a que el profesor licenciado y cualificado con metodologías analógicas enfrenta alumnos considerados nativos digitales. En este sentido, Martins et ál. (2018), Barcelos, Tarouco y Bercht (2009) y Kynäslahti y Seppälä (2003) revelan que la adaptación al uso de móviles en clase y la quiebra de paradigmas tradicionalistas son fundamentales y necesarios para iniciar un proceso de discontinuidad de métodos no atractivos e ineficientes, para dar cabida a herramientas pedagógicas eficientes, productivas, agradables, divertidas y motivadoras para el alumno y el propio docente.

Martins et ál. (2018) y Barreto (2018) ratifican que, en ese proceso de intervención tecnológica en los ambientes escolares, impulsados principalmente por móviles 
como celulares, cámaras y tabletas, el docente afronta la necesidad de entender las posibilidades de cambios ofrecidos por estos dispositivos tecnológicos con respecto a las didácticas y técnicas empleadas con los alumnos. Para Barreto (2018) y Lévy (2011), además del impacto en la escuela y la clase, este nuevo escenario contribuye significativamente a una sociedad más creativa.

A pesar de que la dinámica de los dispositivos móviles se perpetúa en la enseñanza, Martins et ál. (2018) hacen hincapié en la resistencia aparente de muchos profesores. Los autores señalan que ello sucede debido al desconocimiento de las potencialidades del móvil en clase, por no contar con dichos recursos para uso personal, por ser incómodo o por el miedo a lo nuevo. Motivos que también son justificados por Palfrey y Gasser (2011) que caracterizan a los profesores como inmigrantes digitales, ya que no nacieron en la época digital y sí en la analógica.

\section{Modelo de la teoría unificada de aceptación del uso de la tecnología}

El manejo de dispositivos móviles en los últimos años tuvo notorio crecimiento (Ramírez-Correa et ál., 2015). Se sabe que la demanda por servicios de conectividad crecerá exponencialmente y que cada vez más aspectos como conexión, movilidad y ubicuidad (Martins et ál., 2018) estarán presentes en la vida de la gente.

Conforme a los estudios de Venkatesh et ál., (2003), el modelo de la teoría unificada de aceptación del uso de la tecnología (UTAUT) busca medir la aceptación y el uso de esta por parte de los individuos. Se originó por medio de una comparación entre diversos modelos de aceptación y uso de la tecnología. Venkatesh et ál. (2003) los mencionan como la teoría de la acción relacionada (TRA), el modelo de aceptación de la tecnología (TAM), el modelo motivacional (MM), la teoría del comportamiento planeado (ТРв), la combinación entre la ТАм y la ТРв, el modelo de utilización del PC (MPCU), la teoría de la difusión de la innovación (IDT) y la teoría social cognitiva ( $\mathrm{SCT}$ ). A decir de los propios autores, se eligieron a causa de que convergían entre sí y presentaban algunas fallas que impedían pesquisas más complejas sobre el tema. Fueron puestos a prueba en el área de la tecnología de la información, comprobados en la academia y constituidos como referencia en periódicos científicos internacionales.

El modelo utAut, de acuerdo con Venkatesh et ál. (2003), fue desarrollado y construido en seis dimensiones: cuatro independientes, dos dependientes y cuatro vertientes moderadoras (tabla 1). 
Tabla 1. Variables del modelo UTAUT

\begin{tabular}{|l|l|l|}
\hline \multicolumn{1}{|c|}{ Variables independientes } & \multicolumn{1}{c|}{ Variables dependientes } & \multicolumn{1}{c|}{ Variables moderadoras } \\
\hline Expectativa de desempeño & Intención comportamental & Género \\
\hline Expectativa de esfuerzo & Comportamiento al uso & Edad \\
\hline Influencia social & & Experiencia \\
\hline Condiciones facilitadoras & & Vulnerabilidad al uso \\
\hline
\end{tabular}

Fuente: elaboración propia basada en Venkatesh et ál. (2003).

El modelo pretendía medir la aceptación del uso de una determinada tecnología. Sin embargo, Venkatesh, Thong y Xu (2012) advirtieron que estaba siendo utilizado en varios contextos organizacionales y no organizacionales, y en las investigaciones destinadas al consumidor. Por ende, desarrollaron otro más completo, el UTAUT 2, que permitió identificar el comportamiento del consumidor con relación al uso y la intención de uso de las tecnologías. El UTAUT 2, perfeccionado por Venkatesh, Thong y Xu (2012), añadió tres variables a las ya establecidas en el anterior: motivación hedonista, precio/valor y hábito (tabla 2).

Tabla 2. Variables del modelo u TAUT 2.

\begin{tabular}{|l|l|l|}
\hline \multicolumn{1}{|c|}{ Variables independientes } & Variables dependientes & \multicolumn{1}{c|}{ Variables moderadoras } \\
\hline Expectativa de rendimiento & Intención comportamental & Género \\
\hline Expectativa de esfuerzo & Comportamiento al uso & Edad \\
\hline Influencia social & & Experiencia \\
\hline Condiciones facilitadoras & & Vulnerabilidad al uso \\
\hline Motivación hedonista & & \\
\hline Precio/valor & & \\
\hline Hábito & & \\
\hline
\end{tabular}

Fuente: elaboración propia basada en Venkatesh, Thong y Xu (2012). 
El método fue replicado, utilizado y adaptado en varias pesquisas nacionales e internacionales en diferentes contextos y con características peculiares, siempre permeando la intención de uso y aceptación de la tecnología. Por ejemplo: el uso y crecimiento del e-commerce en servicios turísticos asiáticos (Melisa e Indrawati, 2016); la utilización del aplicativo MedFit App por personas que están en rehabilitación cardiovascular (Duff et ál., 2018); la aceptación de la internet móvil por estudiantes universitarios (Ramírez-Correa, 2015); la aceptación de uso de tecnología y su influencia en el manejo de aplicativos móviles por estudiantes (Domingues, Araujo y Alberton, 2018); y la aceptación de uso de Facebook por estudiantes de un distrito rural de Malasia (Raman, Sani y Kaur, 2014). Todo esto pone de manifiesto que independiente de la adaptación del modelo UTAUT 2, la posibilidad de medir la aceptación y el uso de la tecnología rompe barreras y se halla en diversos segmentos.

Específicamente, el estudio de Ramírez-Correa et ál. (2015) tuvo como objetivo validar de forma empírica un modelo de aceptación de dispositivos móviles en estudiantes universitarios brasileños, basado en el modelo utAut 2. Los resultados del análisis indicaron, por un lado, el alto grado de confiabilidad y validez de la medida y, por otro, una relación positiva, fuerte y significativa entre el hábito y la intención de utilizar dispositivos móviles. Además, la intención de utilizar dispositivos móviles está influenciada por el rendimiento esperado, el esfuerzo requerido, la influencia social, la motivación hedónica y las condiciones facilitadoras.

Mariano et ál. (2016) también utilizaron el modelo uTAUT 2 para identificar en estudiantes universitarios la aceptación del uso de aplicaciones en dispositivos móviles y el grado en que cada una afecta la intención de uso. El resultado mostró que todos los factores encontrados entre los que influyen en los estudiantes universitarios obtuvieron un porcentaje de 60,5 \% de correlación con respecto a la intención de utilizar aplicaciones móviles, y que dentro de las dimensiones aquellos que más fueron tomados en consideración y tuvieron mayor relevancia en el público investigador fueron el hábito y la motivación hedonista.

Venkatesh, Thong y Xu (2012) produjeron complementos significativos hasta llegar al modelo utAut 2. Ramírez-Correa et ál. (2015) identificaron las pesquisas y Mariano et ál. (2016) adaptaron el modelo para la utilización de siete variables independientes y apenas una dependiente, usadas en esta indagación y descritas en la figura 1. 


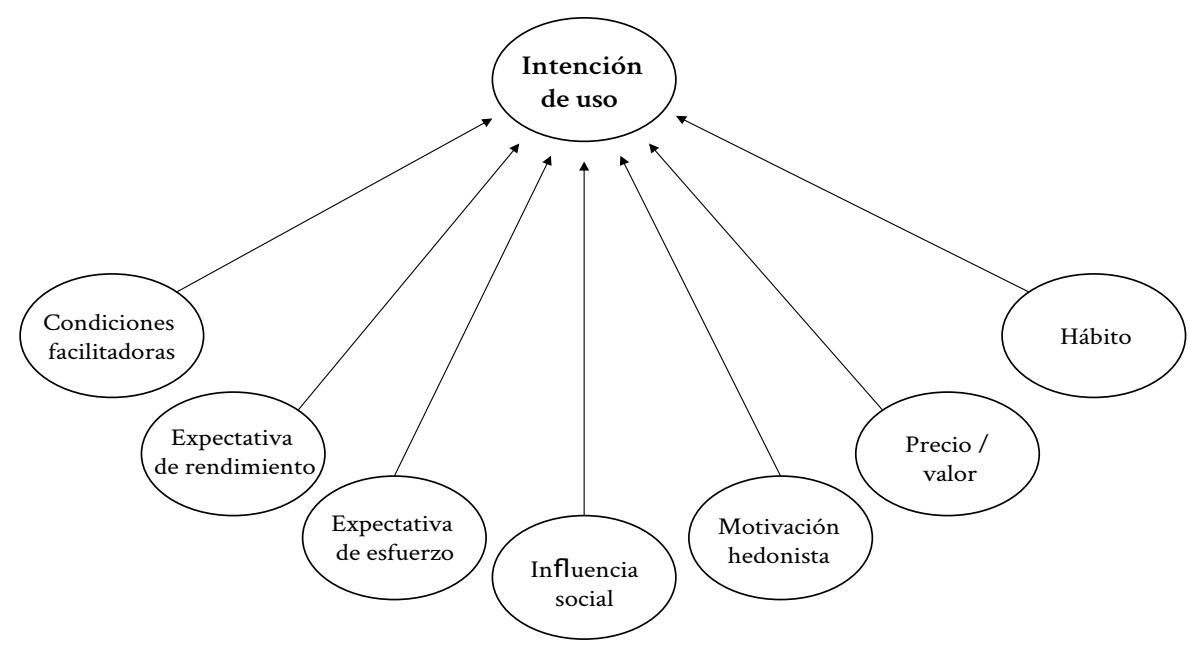

Figura 1. Modelo UTAUT 2.

Fuente: adaptado de Ramírez-Correa et ál. (2015).

A continuación se presentan los procedimientos metodológicos para explicar los conceptos y el surgimiento de las hipótesis de cada dimensión/variable del modelo utAut 2.

\section{Método}

Con vistas a alcanzar los objetivos propuestos, se realizó una investigación cuantitativa de carácter descriptivo. La recolección de información primaria se define como un levantamiento basado en las respuestas de individuos cuyos datos varían en opiniones, actitudes, creencias, etnia, género, renta y edad en gran volumen o cantidad (Hair Jr. et ál., 2005).

Se aplicó un cuestionario on line, auxiliado por Google Docs, ajustado a un conjunto de preguntas según el modelo utaut 2 de Venkatesh, Thong y Xu (2012), adaptado de Ramírez-Correa et ál. (2015), con sus siete variables independientes (condiciones facilitadoras, expectativas de desempeño, influencia social, expectativa de esfuerzo, motivación hedonista, precio/valor y hábito) y una dependiente (intención de uso), con la finalidad de explicar las variables independientes y las dependientes y la construcción de las hipótesis (tablas 3 y 4). Para la aplicación de la recolección de datos se utilizó el modelo UTAUT 2. 
Factores que influyen en el uso de teléfonos móviles en el contexto de aprendizaje...

Tabla 3. Explicación de las variables independientes e hipótesis de investigación.

\begin{tabular}{|c|c|}
\hline $\begin{array}{c}\text { Variables } \\
\text { independientes-siglas }\end{array}$ & Definición constitutiva e hipótesis $(\mathrm{H})$ \\
\hline $\begin{array}{l}\text { Expectativa de rendimiento } \\
\text { (Rendim) }\end{array}$ & $\begin{array}{l}\text { Se refiere a cómo el individuo tiene la posibilidad de mejorar } \\
\text { su rendimiento o desempeño con la utilización de tecnología, } \\
\text { ya sea en el trabajo, en el estudio o en cualquier otra actividad } \\
\text { (Venkatesh et ál., 2003; Raman, Sani y Kaur, 2014; Alves, 2018). } \\
\text { H1: La expectativa de rendimiento influencia positivamente sobre } \\
\text { la intención de uso de móviles por parte del docente. }\end{array}$ \\
\hline $\begin{array}{l}\text { Expectativa de esfuerzo } \\
\text { (Esfor) }\end{array}$ & $\begin{array}{l}\text { De acuerdo con Venkatesh et ál. (2003) y Raman, Sani y Kaur } \\
\text { (2014), el individuo tiene la facilidad de emplear determinada } \\
\text { tecnología. } \\
\text { H2: La expectativa de esfuerzo influencia positivamente sobre la } \\
\text { intención de uso de móviles por el docente. }\end{array}$ \\
\hline $\begin{array}{l}\text { Motivación hedonista } \\
\text { (Hedon) }\end{array}$ & $\begin{array}{l}\text { Venkatesh, Thong e Xu (2012) y Mariano et ál. (2016) entienden } \\
\text { que es la dimensión la que determina cuán placentero es el uso } \\
\text { del móvil para el individuo. Haines (2010), Hills (2008) y Labukt } \\
\text { (2012) destacan el bienestar y el mismo encantamiento que las } \\
\text { personas sienten en el momento del uso del aplicativo. } \\
\text { H3: La motivación hedonista influencia positivamente sobre la in- } \\
\text { tención de uso de móviles por el docente. }\end{array}$ \\
\hline Influencia social (Influen) & $\begin{array}{l}\text { Se refiere a las influencias recibidas por individuos en la } \\
\text { utilización de determinada tecnología móvil. O sea, mide la } \\
\text { influencia; es la interferencia que el individuo siente de personas } \\
\text { próximas para utilizar un aplicativo móvil (Venkatesh et ál., } \\
\text { 2003; Raman Sani y Kaur, 2014; Alves, 2018). } \\
\text { H4: La influencia social incide positivamente sobre la intención de } \\
\text { uso de móviles por el docente. }\end{array}$ \\
\hline $\begin{array}{l}\text { Condiciones facilitadoras } \\
\text { (Facilit) }\end{array}$ & $\begin{array}{l}\text { Las condiciones facilitadoras son definidas por las condiciones } \\
\text { percibidas en la facilidad de uso de determinada tecnología, así } \\
\text { como la base técnica que antecede a la utilización. Es decir: se } \\
\text { refiere a si la tecnología es fácil de utilizar y se posee soporte } \\
\text { técnico delante de algún tipo de problema (Venkatesh et ál., } \\
\text { 2003). } \\
\text { H5: Las condiciones facilitadoras influencian positivamente sobre } \\
\text { la intención de uso de móviles por el docente. }\end{array}$ \\
\hline
\end{tabular}




\begin{tabular}{|c|c|}
\hline $\begin{array}{c}\text { Variables } \\
\text { independientes-siglas }\end{array}$ & Definición constitutiva e hipótesis $(\mathrm{H})$ \\
\hline Precio/valor (Valor) & $\begin{array}{l}\text { En un contexto de uso de tecnología de consumo, el precio se } \\
\text { refiere al coste beneficio de la tecnología empleada y utilizada. } \\
\text { Se relaciona con las expectativas que el individuo percibe en la } \\
\text { utilización del bien (Venkatesh et ál., 2003). } \\
\text { H6: El precio/valor influencia positivamente sobre la intención de } \\
\text { uso de móviles por el docente. }\end{array}$ \\
\hline Hábito (Habito) & $\begin{array}{l}\text { Se refiere a la familiaridad en la utilización, así como a las } \\
\text { acciones automáticas o condicionadas al uso de determinada } \\
\text { tecnología (Venkatesh, Thong y Xu, 2012; Mariano et ál., 2016). } \\
\text { H7: El hábito influencia positivamente sobre la intención de uso } \\
\text { de móviles por el docente. }\end{array}$ \\
\hline
\end{tabular}

Fuente: elaboración propia.

La definición constitutiva de la variable dependiente (intención de uso) o modelo UTAUT 2 se resume en la tabla 4 .

Tabla 4. Explicación de la variable dependiente de investigación.

\begin{tabular}{|c|c|}
\hline $\begin{array}{l}\text { Variable } \\
\text { dependiente-sigla }\end{array}$ & Definición constitutiva \\
\hline Intención de uso (Intuso) & $\begin{array}{l}\text { Esta variable es resultado de las influencias ocasionadas por } \\
\text { las dimensiones o variables independientes, lo que motiva la } \\
\text { identificación del comportamiento del consumidor con relación } \\
\text { al uso y a la intención de uso de las tecnologías (Venkatesh, } \\
\text { Thong y Xu, 2012). }\end{array}$ \\
\hline
\end{tabular}

Fuente: elaboración propia.

Los datos fueron recolectados y medidos con la ayuda de la escala Likert, que brinda la posibilidad de presentar variables entre categorías de respuestas: entre "en desacuerdo total” (1) y “de acuerdo total” (7). Aquí los participantes debían indicar el grado de concordancia o discordancia con arreglo a cada cuestionamiento.

Los encuestados corresponden a docentes de la enseñanza superior de facultades, centros universitarios y universidades de la provincia de Santa Catarina, al sur 
de Brasil. La muestra estuvo integrada por 158 interlocutores de 301 solicitaciones. De los 158 cuestionarios respondidos se obtuvieron 134 cuestionarios respondidos. Cabe mencionar que se realizó un análisis unidimensional debido a seis ítems faltantes en las respuestas, que se rellenaron mediante los promedios de la dimensión. Después se llevó a cabo el análisis de outliers, por lo cual hubo que descartar a 24 de los que, habiendo contestado, dejaron de rellenar más del $90 \%$ de los temas.

Se identificaron siete hipótesis denominadas $\mathrm{H} 1$ y $\mathrm{H} 7$ que posibilitaron esclarecer y constatar ideas y orientar a los investigadores en la conducción del estudio. La indagación podría catalogarse de corte transversal (Hair Jr. et ál., 2005), considerando que la recolección de información sucedió en apenas un periodo de tiempo, entre el 9 de agosto y el 5 de septiembre de 2018.

La tabulación de los datos, el análisis de la estadística descriptiva, la correlación y la regresión lineal múltiple se efectuó mediante el software estadístico spss for Windows, versión 21, lo que facilitó verificar la influencia de múltiples variables independientes sobre una o más variables dependientes (Hair Jr. et ál., 2005).

\section{Resultados}

\section{Presentación de resultados}

Se identificó inicialmente el perfil de la muestra seleccionada (134 interlocutores) y la correlación entre las dimensiones en la secuencia analizada. Se utilizó el método de regresión lineal múltiple, con el fin de verificar la carga de cada dimensión variable independiente sobre la dependiente y las respectivas significancias (p-valor) (tabla 5).

Tabla 5. Análisis descriptivo de la muestra investigada.

\begin{tabular}{|c|c|c|c|c|c|c|c|c|}
\hline \multicolumn{2}{|c|}{ Género } & \multicolumn{2}{c|}{$\begin{array}{c}\text { Tiempo de } \\
\text { docencia }\end{array}$} & \multicolumn{3}{c|}{ Titulación } & \multicolumn{2}{c|}{$\begin{array}{c}\text { Sistema } \\
\text { operacional }\end{array}$} \\
\hline Masculino & Femenino & $\begin{array}{c}<15 \\
\text { años }\end{array}$ & $\begin{array}{c}>15 \\
\text { años }\end{array}$ & Espec. & Máster & Doctor & Android & iPhone \\
\hline $64 \%$ & $36 \%$ & $83 \%$ & $17 \%$ & $47 \%$ & $29 \%$ & $24 \%$ & $71 \%$ & $29 \%$ \\
\hline
\end{tabular}

Fuente: elaboración propia. 
El análisis preliminar mostró que el $64 \%$ de interlocutores son del género masculino, con menos de 15 años en la docencia, con escolarización lato sensu, y utilizan como sistema operacional Android. La caracterización se hizo tomando en cuenta la correlación entre las dimensiones, y así se logró revelar la fuerza y la dirección del relacionamiento lineal entre las variables. Tal y como detalla la tabla 6, hay Habito, Valor e Intuso sobre la influencia de intención de uso de móviles en la sala de clase por parte del docente.

Tabla 6. Resultado de la correlación entre las variables independientes encuestadas.

\begin{tabular}{|c|c|c|c|c|c|c|c|c|}
\hline & & Rendim & Esfor & Influen & Facilit & Hedon & Valor & Habito \\
\hline \multirow{2}{*}{ Esfor } & Correlación & $0,604^{* *}$ & & & & & & \\
\hline & Significancia & 0,000 & & & & & & \\
\hline \multirow{2}{*}{ Influen } & Correlación & $0,395^{* *}$ & $0,213^{*}$ & & & & & \\
\hline & Significancia & 0,000 & 0,013 & & & & & \\
\hline \multirow{2}{*}{ Facilit } & Correlación & $0,516^{* *}$ & $0,553^{* *}$ & $0,377^{* *}$ & & & & \\
\hline & Significancia & 0,000 & 0,000 & 0,000 & & & & \\
\hline \multirow{2}{*}{ Hedon } & Correlación & $0,465^{* *}$ & $0,381^{* *}$ & $0,212^{*}$ & $0,434^{* *}$ & & & \\
\hline & Significancia & 0,000 & 0,000 & 0,014 & 0,000 & & & \\
\hline \multirow{2}{*}{ Valor } & Correlación & $0,357^{* *}$ & $0,225^{* *}$ & $0,232^{* *}$ & $0,303^{* *}$ & $0,401^{* *}$ & & \\
\hline & Significancia & 0,000 & 0,009 & 0,007 & 0,000 & 0,000 & & \\
\hline \multirow{2}{*}{ Habito } & Correlación & $0,604^{* *}$ & $0,410^{* *}$ & $0,458^{* *}$ & $0,385^{* *}$ & $0,445^{* *}$ & $0,589^{* *}$ & \\
\hline & Significancia & 0,000 & 0,000 & 0,000 & 0,000 & 0,000 & 0,000 & \\
\hline \multirow{2}{*}{ Intuso } & Correlación & $0,662^{* *}$ & $0,512^{* *}$ & $0,403^{* *}$ & $0,492^{* *}$ & $0,570^{* *}$ & $0,687^{* *}$ & $0,738^{* *}$ \\
\hline & Significancia & 0,000 & 0,000 & 0,000 & 0,000 & 0,000 & 0,000 & 0,000 \\
\hline
\end{tabular}

Fuente: elaboración propia. 
La dimensión hábito presentó una correlación de 0,738, inherente al constructo (Habito). Presume una alta correlación positiva con la intención de aceptación de uso de móviles por parte de los docentes en el aula, al igual que la dimensión precio/valor, con el 68,7 \% de intención de uso. La dimensión expectativa de rendimiento (Rendim), con una fuerza de asociación 0,662, influencia la intención de uso de móviles. La tabla 7 o R ${ }^{2}$ (R cuadrado) ilustra la variación de explicación del modelo, el cual demuestra que las variables o dimensiones independientes explican el $75 \%$ del modelo estudiado referente a la intención de uso de móviles en clase por parte de los docentes.

Tabla 7. Resultado de la regresión del modelo investigado.

\begin{tabular}{|c|c|c|c|c|}
\hline Modelo & $\mathbf{R}$ & $\mathbf{R}^{2}$ & $\mathbf{R}^{2}$ ajustado & $\begin{array}{c}\text { Error estándar } \\
\text { de la estimativa }\end{array}$ \\
\hline 1 & $0,866^{\mathrm{a}}$ & 0,750 & 0,736 & 0,5074 \\
\hline A = Predictores: (Constante), Habito, Facilit, Influen, Hedon Esfor, Valor, Rendim \\
\hline
\end{tabular}

Fuente: elaboración propia.

La prueba de regresión lineal múltiple, según lo sintetizado en la tabla 8 , busca identificar cuál variable tiende a influenciar la intención del uso de aplicaciones móviles por parte de los docentes de la enseñanza superior en clase.

Tabla 8. Regresión lineal múltiple.

\begin{tabular}{|c|c|c|c|c|c|}
\hline \multirow{2}{*}{ Modelo } & \multicolumn{2}{|c|}{$\begin{array}{l}\text { Coeficientes no } \\
\text { estandarizados }\end{array}$} & \multirow{2}{*}{$\begin{array}{c}\begin{array}{c}\text { Coeficientes } \\
\text { estandarizados }\end{array} \\
\text { Beta }\end{array}$} & \multirow{2}{*}{$\mathbf{t}$} & \multirow{2}{*}{$\begin{array}{c}\text { Sig. } \\
\text { (p-valor) }\end{array}$} \\
\hline & B & Modelo estándar & & & \\
\hline (Constante) & $-1,676$ & 0,503 & & $-3,331$ & 0,0030 \\
\hline Rendin & 0,302 & 0,100 & 0,203 & 3,030 & 0,0475 \\
\hline Esfor & 0,142 & 0,071 & 0,121 & 2,001 & 0,2823 \\
\hline Influen & 0,053 & 0,049 & 0,057 & 1,080 & 0,6337 \\
\hline Facilit & 0,040 & 0,085 & 0,028 & 0,478 & 0,0066 \\
\hline Hedon & 0,164 & 0,059 & 0,150 & 2,761 & 0,0000 \\
\hline
\end{tabular}




\begin{tabular}{|l|c|c|c|c|c|}
\hline \multirow{2}{*}{ Modelo } & \multicolumn{2}{|c|}{$\begin{array}{c}\text { Coeficientes no } \\
\text { estandarizados }\end{array}$} & $\begin{array}{c}\text { Coeficientes } \\
\text { estandarizados }\end{array}$ & t & $\begin{array}{c}\text { Sig. } \\
\text { (p-valor) }\end{array}$ \\
\cline { 2 - 6 } & B & Modelo estándar & Beta & 6,289 & 0,0003 \\
\hline Valor & 0,405 & 0,064 & 0,357 & 3,688 & 0,0030 \\
\hline Habito & 0,235 & 0,064 & 0,252 & & \\
\hline A = variable dependiente: Intuso
\end{tabular}

Fuente: elaboración propia.

Los datos de la tabla 8 avalan que cinco dimensiones poseen significancia conforme p-valor: expectativa de rendimiento (Rendin-0,0475), condiciones facilitadoras (Facilit-0,0066), motivación hedonista (Hedon-0,000), precio/valor (Valor-0,0003) y hábito (Habito-0,0030).

Así mismo, se observó la carga de relación con la variable dependiente intención de uso (Intuso): precio/valor (Valor-0,357), expectativa de rendimiento (Rendin-0,203), hábito (Habito-0,252), motivación hedonista (Hedon-0,150) y expectativa de esfuerzo (Esfor-0,121). Pese a que la dimensión Esfor presenta carga de 0,121, su significancia es baja $(0,2823)$, y en este caso no manifiesta evidencia y consecuente soporte para configurar la hipótesis 2 (H2), donde la expectativa de esfuerzo influencia positivamente sobre la intención de uso de móviles por parte del docente.

\section{Análisis y discusión de resultados}

Respecto a la dimensión expectativa de rendimiento (Rendin), se advierte que los docentes creen que los móviles influencian positivamente el rendimiento de las actividades en la sala de clase (Venkatesh et ál., 2003; Raman, Sani y Kaur, 2014; Alves, 2018), debido a que pueden ser utilizados en cualquier lugar y momento, de acuerdo con lo dicho por Mariano et ál. (2016). Así, la intención de aceptación da soporte para confirmar la H1, al observar la carga de relación $(0,23)$ y el p-valor de 0,0475. Consecuentemente, "La expectativa de rendimiento influencia positivamente sobre la intención de uso de móviles por el docente” se aprecia en esta investigación.

Se identificaron dos dimensiones que no presentaron significancias suficientes en los análisis. En primer lugar, se encuentra la expectativa de esfuerzo (Esfor), en referencia a la facilidad del individuo para la utilización de determinada tecnología, tal y como lo diagnostican Venkatesh et ál. (2003) y Raman, Sani y Kaur (2014). En este 
caso, no hay evidencias que sostengan la H2. "La expectativa de esfuerzo influencia positivamente sobre la intención de uso de móviles por el docente” presentó p-valor de 0,2823 . Esto indica que los individuos no muestran preocupación relevante sobre el desempeño del aplicativo, según los estudios de Mariano et ál. (2016).

La dimensión Hedon, que presentó carga de relación con la variable dependiente de 0,150 y p-valor de 0,00, se ha concebido como una dimensión que influencia la intención de uso de móviles en esta investigación, como en las pesquisas realizadas por Venkatesh, Thong y Xu (2012), Mariano et ál. (2016) y Ramírez-Correa et ál. (2015). Intuyendo que la motivación hedonista atañe al placer y a la satisfacción (Haines, 2010; Hills, 2008; Labukt, 2012) de utilizar un móvil, es posible advertir que los docentes prestan intención de uso, dado el despertar aparente de la sensación de bienestar y de las emociones positivas que proporciona. Por lo tanto, se confirma la H3: "La motivación hedonista influencia positivamente sobre la intención de uso de móviles por el docente".

La segunda hipótesis que no fue considerada es la dimensión influencia social (Influen). Dicha variable, según Venkatesh et ál. (2003), Raman, Sani y Kaur (2014) y Alves (2018), está alineada con las influencias recibidas de personas que se relacionan y que son importantes para el individuo que utiliza determinada aplicación móvil. Para los docentes, la influencia u opinión de las personas con las que conviven tiende a ejercer poca influencia en la intención de uso de móviles. De esa manera, la H4, al referir que "La influencia social incide positivamente sobre la intención de uso de móviles por parte del docente", no presenta evidencias suficientes para confirmarla.

Por su parte, condiciones facilitadoras (Facilit) presentó baja carga de relación con la variable dependiente $(0,028)$. Con todo, se identificó significancia que soporta la H5: "Las condiciones facilitadoras influencian positivamente sobre la intención de uso de móviles por el docente”. Venkatesh et ál., (2003) plantean que esta variable alude a las facilidades de uso de determinada tecnología vivenciada por el individuo. Los docentes creen en las facilidades de acceder y manipular móviles en clase.

La dimensión Valor, además de presentar carga de relación con la variable dependiente (Intuso), también demostró significancia de 0,003. Se consigue, entonces, soportar la H6, que sugiere: "El precio/valor influencia positivamente sobre la intención de uso de móviles por el docente”. Esto ratifica lo expresado por Venkatesh, Thong y Xu (2012) y Venkatesh et ál. (2003), cuando afirman que en la intención de uso de determinada tecnología en un contexto de consumo el precio está relacionado con el coste-beneficio. 
En la dimensión hábito, su carga de relación $(0,252)$ y p-valor de 0,003 confirman la H7: "El hábito influencia positivamente sobre la intención de uso de móviles por el docente”. Venkatesh, Thong y Xu (2012), Mariano et ál. (2016) y Ramírez-Correa et ál. (2015) puntualizan que el hábito de utilizar una aplicación móvil en las actividades diarias es automático, una vez que el individuo está condicionado y familiarizado con los recursos tecnológicos.

Dado el contexto planteado, la siguiente tabla comparativa expone los resultados obtenidos bajo el mismo modelo (UTAUt 2). Además, puntea los factores que influencian la intención de uso de móviles en los estudios de Ramírez-Correa et ál. (2015) y Mariano et ál. (2016).

Tabla 9. Comparación de resultados entre estudios.

\begin{tabular}{|c|c|c|c|c|}
\hline Variable & Dimensiones & $\mathbf{A}^{1}$ & $\mathbf{A}^{2}$ & $\mathbf{A}^{3}$ \\
\hline Expectativa de rendimiento & Rendim & $\mathrm{X}$ & $\mathrm{x}$ & \\
\hline Expectativa de esfuerzo & Esfor & & & \\
\hline Influencia social & Influen & & $\mathrm{X} 4$ & \\
\hline Condiciones facilitadoras & Facilit & $\mathrm{X}$ & $\mathrm{X} 4$ & \\
\hline Motivación hedonista & Hedon & $\mathrm{X}$ & & $\mathrm{X}$ \\
\hline Precio/valor & Valor & $\mathrm{X}$ & & \\
\hline Hábito & Habito & $\mathrm{X}$ & $\mathrm{X}$ & $\mathrm{X}$ \\
\hline \multicolumn{5}{|c|}{$\begin{array}{l}\text { Notas: } \mathrm{A}^{1}=\text { resultado de los autores; } \mathrm{A}^{2}=\text { resultados de Ramírez-Correa et ál. (2015); } \\
\mathrm{A}^{3}=\text { resultados de Mariano et ál. (2016); X4 = resultados con menor influencia de Ramírez- } \\
\text { Correa et ál. (2015) }\end{array}$} \\
\hline
\end{tabular}

Fuente: elaboración propia.

Cabe resaltar que la tabla 9 refleja una comparación de resultados mediante la utilización del modelo utAut 2, teniendo en cuenta que Ramírez Correa et ál. (2015) y Mariano et ál. (2016) llevaron a cabo su pesquisa de acuerdo con los criterios de académicos universitarios, y en este trabajo, con base en lo que respondieron docentes de la enseñanza superior. En sus estudios la expectativa de rendimiento y el hábito son los factores que más influencian en la intención de uso de móviles. Los autores opinan que la expectativa de rendimiento corresponde al nivel en que la tecnología puede 
mejorar el desempeño de las personas en el trabajo o en los estudios (Rendin); y el hábito (Habito) forma parte del uso de un público en su mayoría joven, que entiende que esta herramienta lo auxilia en sus actividades cotidianas, por ejemplo, acciones orientadas al uso de redes sociales.

Mariano et ál. (2016) consideran que los factores que más han influenciado fueron hábito y motivación hedonista, respectivamente, lo que corrobora los resultados de Ramírez-Correa et ál. (2015). Mariano et ál. (2016) explican que una posible justificación sería el promedio de edad (22,52 años), en razón de que este perfil de usuario tiende a dar mayor importancia a ese tipo de dispositivo móvil en las actividades interpersonales diarias (Habito), además de que trae una sensación de placer y satisfacción al usarlo (Hedon).

\section{Consideraciones finales}

El trabajo tuvo como objetivo evaluar los factores que influyen en el uso de teléfonos móviles en el contexto del aprendizaje de los docentes de educación superior. Para ello se apeló al modelo utAut 2, adaptado de Ramírez-Correa et ál. (2015), con sus siete variables independientes (condiciones facilitadoras, expectativas de desempeño, influencia social, expectativa de esfuerzo, motivación hedonista, precio/valor y hábito) y una dependiente (intención de uso).

Los datos se recolectaron mediante la implementación de un cuestionario a docentes de la educación superior de la provincia de Santa Catarina, sur de Brasil, y se analizaron por medio de las técnicas de correlación. Esto puso a prueba la fuerza y la dirección del relacionamiento lineal entre variables y de regresión, con el objetivo de determinar cuáles tienden a influenciar la intención del uso de aplicaciones móviles (mobiles) por parte de los docentes en clase.

Los resultados indican que el hábito, el precio/valor y la expectativa de rendimiento poseen mayor fuerza de asociación sobre la variable dependiente. Otras dimensiones que también se vieron en el análisis de regresión lineal, ya que apuntan hacia cuáles factores influencian la intención de uso de móviles en la sala de clase por parte del docente, fueron: precio/valor, expectativa de rendimiento, hábito, motivación hedonista, condiciones facilitadoras. Vale destacar que al recurrir a la prueba R2, se observó que $75 \%$ del modelo estudiado está explicado con la técnica de regresión. Esto implica que esos factores en conjunto pueden explicar cerca del 75 \% de la intención de uso de las aplicaciones móviles por los docentes. 
La investigación corrobora parcialmente los estudios de Venkatesh, Thong y Xu (2012) y Mariano et ál. (2016), al reconocer las variables precio/valor, hábito y motivación hedonista como factores influyentes también en este trabajo. La similitud observada con la investigación de Ramírez Correa et ál. (2015) fueron los factores de expectativa de rendimiento, hábito y condiciones facilitadoras.

Ramírez-Correa et ál. (2015) señalaron un factor más que incide en la intención de uso de móviles y que tiene que ver con la influencia social. Esta dimensión no tuvo significancia. Una posible explicación se basa en el perfil de los encuestados, en su mayoría (87 \%) con 15 años en la docencia. Es decir, cuentan con una madurez hipotéticamente constituida, debido al grado de instrucción, la edad y la vivencia profesional. Este docente difícilmente puede ser influenciado por personas de su ambiente a usar o no determinadas tecnologías móviles, visto que, de las 134 respuestas válidas, el 95,52 \% utilizan móviles en clase hace más de 12 meses.

Desde el punto de vista teórico, se espera que la investigación contribuya tanto a una mayor aceptación como a la utilización más frecuente de la herramienta UTAUT 2 , constituida como referencia en estudios métricos sobre la intención de uso de tecnologías. Cuando se tratan aspectos empíricos y gerenciales, les corresponde a los docentes interiorizar cada vez más la necesidad del uso de tecnologías en clase, una vez que el entorno universitario ha provocado cambios en sus vertientes pedagógicas y en el proceso de enseñanza-aprendizaje.

Como limitaciones podrán mencionarse la periodicidad y la circunscripción de la muestra solo a docentes universitarios de la provincia de Santa Catarina, en virtud de que estos ejercen por al menos 15 años. Este pudo haber sido un factor que tendiera a una mayor influencia de las variables precio/valor, expectativa de rendimiento, hábito, motivación hedonista y condiciones facilitadoras. Se sugiere la aplicación del modelo teórico utaut 2 en futuros estudios, en los que participen docentes de todo el territorio nacional, con la finalidad de comparar e identificar resultados por región, y que sirvan como herramienta de acción de apoyo a los gobiernos y a las instituciones comprometidas con el proceso educativo.

\section{Sobre los autores}

Maria José Carvalho de Souza Domingues. Doctora en Ingeniería de Producción y magíster en Administración Pública por la Universidad Federal de Santa Catarina (UFSC), Brasil. Líder del Grupo de Investigación de Organizaciones Complejas y Educación Superior del Consejo Nacional de Desarrollo 
Factores que influyen en el uso de teléfonos móviles en el contexto de aprendizaje...

Científico y Tecnológico (CNPQ), Brasil. Profesora titular del programa de Posgrado en Administración y Contabilidad de la Universidad Regional de Blumenau, Santa Catarina, Brasil. Ha liderado proyectos de investigación como investigadora principal, y actualmente es coinvestigadora del proyecto "Recursos competitivos de las universidades desde la perspectiva de sus stakeholders", financiado por CNPq/Brasil. Sus publicaciones más recientes son el libro Administração Universitária: temas emergentes (2017), el capítulo de libro “A Educação superior em Santa Catarina” (2018), y dos artículos publicados en el 2020: "Brand equity e o uso de tecnologias da informação e da comunicação aplicadas ao ensino superior: a influência da utilização frente à percepção de marca da comunidade acadêmica de SC" (2020) y “O Hedonismo De Alunos Universitários E Suas Consequências”. Investigadora en temas relacionados con gestión universitaria, toma de decisiones universitarias y tecnologías digitales en educación superior.

Péricles Ewaldo Jader Pereira. Magíster en Administración-Gestión, Internacionalización y Logística por la Universidad de Vale do Itajaí (Univali) (2017). Doctorando en Contabilidad y Administración por la Universidad Regional de Blumenau (FURB). Especialista en Hub Management por Uniasselvi (2019), en Business and Sales Management por Uniasselvi (2015), en Business Marketing por Uniasselvi (2015) у мBA en Liderazgo y Coaching por Uniasselvi (2016). Licenciado en Administración (Tecnología en Procesos de Gestión) por la Sociedad Educativa de Santa Catarina (2014). Tiene experiencia en administración, con énfasis en dirección de empresas y marketing. Tutor de disciplinas en el Centro Universitario Leonardo da Vinci (Uniasselvi/NEAD). Docente de docencia presencial y estudios de posgrado en el Centro Universitario Leonardo da Vinci (Uniasselvi).

Jéferson Deleon Fávero. Magíster en Administración por la Universidad Regional de Bluemanu (FURB), Blumenau, Brasil. Doctorando en Contabilidad y Administración (PPGCCA-FURB). Tiene una especialización lato sensu en Gestión Financiera y de Costos, en Dirección de Empresas, y en Gestión y Mentoría, por el Instituto de Posgrados de Santa Catarina (ICPG). Graduado en Administración con énfasis en finanzas, del Centro Universitario Leonardo da Vinci (Uniasselvi). Actualmente es coordinador de operaciones en SESI/SENAI, Vale do Itajaí, SC, y profesor de gestión financiera y logística (Uniasselvi, Indaial). Tiene experiencia en administración, enfocado en finanzas y logística. Sus líneas de investigación son gestión organizacional y logística.

\section{Referencias}

Alves, D. C. L. (2018). Uso de novas tecnologias na sala de aula: percepção dos professores. CIET: EnPED, 1-7. Recuperado de https://cietenped.ufscar.br/submissao/index. $\mathrm{php} / 2018 /$ article/download/246/291

Angeluci, A. C. B., Soares, S. C. y De Azevedo, A. B. (2018). Usos e apropriações dos celulares: uma perspectiva educacional. Educação y Linguagem, 21(1), 23-40. https://doi. org/10.15603/2176-1043/el.v21n1p23-40 
Arruda, D. E. P. y Puentes, R. V. (2011). A docência no ensino superior: a formação de professores para atuar com tecnologias na educação presencial e a distância. Revista Ensino em Re-Vista, 18(2), 247-258.

Barcelos, R., Tarouco, L. y Bercht, M. (2009). O uso de mobile learning no ensino de algoritmos. Renote, 7(3), 327-337. https://doi.org/10.22456/1679-1916.13573

Barreto, R. P. (2018). Dispositivos móveis digitais e desafios da pesquisa e ensino-aprendizagem de escrita e leitura on-lines. Interfaces Cientificas-Educação, 6(3), 97-106. https://doi. org/10.17564/2316-3828.2018v6n3p97-106

Bautista, G., Borges, F. y Flores, A. (2010). Didáctica universitária en entornos virtuales de enseñanza-aprendizaje. Madrid: Narcea.

Coll, C. y Monereo, C. (2010). Psicologia da educação virtual: aprender a ensinar com as tecnologias da informação e da comunicação. Porto Alegre: Artmed.

Domingues, M. J. C de S., Araújo, B. A. G. G. y Alberton, M. M. (2018). Fatores de aceitação de uso da tecnologia e sua influência na utilização de aplicativos moveis pelos estudantes de ciências contábeis. XII Congresso Anpcont, 1-13. Recuperado de http://anpcont.org.br/ pdf/2018_EPC477.pdf

Duff, O., Walsh, D., Malone, S., McDermott, L., Furlong, B, O'Connor, N., et ál. (2018). MedFit app, a behavior-changing, theoretically informed mobile app for patient self-management of cardiovascular disease: user-centered development. Jmir Formative Research, 2(1) 1-13. http://doi.org/10.2196/formative.9550

Fernandes, K. T., da Trindade, G. O., Rêgo, A. H. G., de Miranda, L. C., Lucena, M. J. N. R. y Gomes, A. V. (2012). E-learning via dispositivos móveis no Brasil: Estado da arte e desafios à luz do acesso participativo e universal do cidadão brasileiro ao conhecimento. Anais do Workshop de Desafios da Computação Aplicada à Educação, 128-137. Recuperado de https:// www.br-ie.org/pub/index.php/desafie/article/view/2783/2436

Gomes, M.J. (2008). Na senda da inovação tecnológica na educação a distância. Revista Portuguesa de Pedagogia, 42(2), 181-202. https://doi.org/10.14195/1647 -8614_42-2_10

Haines, W. A. (2010). Hedonism and the variety of goodness. Utilitas, 22(2), 148-170.

Hair Jr, J. F., Babin, B., Money, A. y Samouel, P. (2005). Fundamentos de métodos de pesquisa em administração. Porto Alegre: Bookman.

Hills, A. (2008). Value, reason and hedonism. Utilitas, 2O(1), 50-58.

Humanante-Ramos, P., García-Peñalvo, F. J. y Conde-González, M. (2017). Entornos personales de aprendizaje móvil: una revisión sistemática de la literatura. RIED. Revista Iberoamericana de Educación a Distancia, 20(2), 73-92.

Imbernón, F. (2011). Formação docente e profissional: formar-se para a mudança e a incerteza. São Paulo: Cortez.

Kynäslahti, H. y Seppälä, P. (2003). Professional mobile learning. Helsinki: IT Press. 
Factores que influyen en el uso de teléfonos móviles en el contexto de aprendizaje...

Labukt, I. (2012). Hedonic tone and the heterogeneity of pleasure. Utilitas, 24(2), 172-199. https://doi.org/10.1017/S0953820812000052

Lévy, P. (2011). A inteligência coletiva: por uma antropologia do ciberespaço. $8^{\circ}$ ed. São Paulo: Ediçoes Loiola.

Marçal, E., Andrade, R. y Rios, R. (2005). Aprendizagem utilizando dispositivos móveis com sistemas de realidade virtual. Renote, 3(1), 1-11. https://doi.org/10.22456/1679-1916.13824

Mariano, A., Moraes, J. T. L., Coutinho, G. T. B. y Azar, M. (2016). Fatores determinantes da utilização de aplicativos em dispositivos móveis: um estudo da percepção de estudantes universitários. XL EnANPAD 2016, 1-20. Costa do Sauípe, BA.

Martins, J. V. G., Menezes, R. M. T., Terçariol, A. A. L., Gitahy, R. R. C. y Ikeshoji, E. A. B. (2018). O uso de dispositivos móveis na sala de aula: pedagogia de projetos e tecnologias móveis na educação superior. Revista Ibero-Americana de Estudos em Educação, 13(2), 500-519. https://doi.org/10.21723/riaee.nesp1.v13.2018.10047

Matoski, A., Veiga, B. P., da Silva, M. T. Q. S., Ribeiro, D. G. F. y Alberti, M. E. (2020). Uso de dispositivos móveis como ferramenta de aprendizado: riscos e oportunidades/Use of mobile devices as a learning tool: risks and opportunities. Brazilian Journal of Development, 6(1), 4673-4687. https://doi.org/10.34117/bjdv6n1-335

Medeiros, Z. y Ventura, P. C. S. (2007). O conceito cultura tecnológica e um estudo no meio educacional. Ensaio Pesquisa em Educação em Ciências, 9(2), 237-251.

Melisa, M. y Indrawati, I. (2016). Analysis of customer accep tance of online travel agent technology using UTAUT 2 model (a case study on e-commerce traveloka in indonesia). In Sustainable Collaboration in Business, Technology, Information and Innovation (SCBTII) ( $\mathrm{p}$. 115). Bandung, Indonesia.

Palfrey, J. y Gasser, U. (2011). Nascidos na era digital: entendendo a primeira geração de nativos digitais. Porto Alegre, RS: Artmed.

Pina, F., Kurtz, R., Ferreira, J. B., Freitas, A., Silva, J. F. D. y Giovannini, C. J. (2016). Adoção de m-learning no ensino superior: o ponto de vista dos professores. REAd. Revista Eletrônica de Administração, 22(2), 279-306. https://doi.org/10.1590/1413-2311.0262015.54352

Raman, A., Sani, R. M. y Kaur, P. (2014). Facebook as a collaborative and communication tool: a study of secondary school students in Malaysia. Procedia-Social and Behavioral Sciences, 155(sup. C), 141-146. https://doi.org/10.1016/j.sbspro.2014.10.270

Ramírez-Correa, P., Mariano, A. M., Alfaro-Perés, J. y Marion, M. R. (2015). Aceptación de internet móvil en estudiantes universitarios brasileños: un estudio empírico usando modelado de ecuaciones estructurales. Espacios, 36(13), 1-13.

Rocha, S. S. D., Joye, C. R. y Moreira, M. M. (2020). A educação a distância na era digital: tipologia, variações, uso e possibilidades da educação online. Research, Society and Development, 9(6). http://dx.doi.org/10.33448/rsd-v9i6.3390

Torrezan, C. A. W, y Behar, P. A. (2009). Parâmetros para a construção de materiais educacionais digitais do ponto de vista do design pedagógico. Porto Alegre: Artmed. 
Traxler, J. (2011). Mobile learning: starting in the right place, going in the right direction? International Journal of Mobile and Blended Learning, 3(2), 57-67.

Unesco. (2013). Diretrizes de políticas para a aprendizagem móvel. París. Recuperado de http:// unesdoc.unesco.org/images/0022/002277/227770por.pdf

Venkatesh, V., Morris, M. G., Davis, G. B. y Davis, F. D. (2003). User acceptance of information technology: toward a unified view. MIS Quarterly, 27(3), 425-478. https://doi. org $/ 10.2307 / 30036540$

Venkatesh, V., Thong, J. Y. L. y Xu, X. (2012). Consumer acceptance and use of information technology: extending the unified theory of acceptance and use of technology. MIs Quarterly, 36(1), 157-178. https://doi.org/10.2307/41410412 